\title{
The role of vertical disparity in the scaling of stereoscopic depth perception: An empirical and theoretical study
}

\author{
BARBARA GILLAM \\ University of New South Wales, Kensington, Australia \\ DEBORAH CHAMBERS \\ New School for Social Research, New York, New York \\ and \\ BO LAWERGREN \\ Hunter College of the City, New York, New York \\ and University of New York, New York, New York
}

\begin{abstract}
The present study investigated the possible ecological role of vertical disparity. Specifically, the stereoscopic slant response to vertical magnification of one eye's view (i.e., the induced effect) was measured as a function of degree of magnification and observation distance. As a control, the stereoscopic response to equivalent amounts of horizontal disparity (i.e., the geometric effect) was measured under the same conditions. Unlike the geometric effect, the induced effect did not vary with observation distance, and at all distances it reached an asymptote at approximately $2 \%$ magnification. There was evidence that at very low magnification values the induced effect was greater than the geometric effect. These data are discussed in relation to theories in which the stereoscopic effect of vertical disparity is attributed to the process by which horizontal disparity is scaled for surface eccentricity and distance. The data are consistent with such a theory only if the assumption is made that the scaling effect of vertical disparity is attenuated by conflicting indicators of eccentricity given by convergence. It is argued that direct computational theories, which do not postulate an explicit representation of eccentricity or distance as part of the scaling process, cannot account for the differences obtained between the magnitude of the induced effect and that of the geometric effect.
\end{abstract}

The binocular induced effect is an apparent slant around a vertical axis caused by vertical disparity. It occurs when one eye's image is subject to vertical magnification by an afocal lens (Ogle, 1950). The slant is opposite in direction to the geometric effect, which results from horizontal magnification of one eye's image. The induced effect is regarded as somewhat mysterious since vertical disparity, unlike horizontal disparity, would not normally result when a surface is slanted.

One obvious way to study the induced effect (Arditi, Kaufman, \& Movshon, 1981) is to examine the effect of vertical magnification on orientation disparity, which is a geometric stimulus for depth perception. Figure 1 shows that the orientation disparities produced by vertical mag-

This work was supported by National Science Foundation Grant BNS 8311613 and carried out while the authors were at the Schnurmacher Institute, State University of New York, State College of Optometry. The writing was carried out while the senior author was at Oxford University on a Guggenheim Fellowship. She wishes to thank Brian Rogers for helpful discussions. Correspondence concerning this article should be addressed to Barbara Gillam at the School of Psychology, University of New South Wales, P.O. Box 1, Kensington, N.S.W., Australia 2033. nification of one image of several nonvertical lines are indeed consistent with a tilt of the lines in depth, but that these tilts do not cumulatively result in a planar surface for lines of different orientation. It seems that at some degree of stimulus complexity, a planar solution, in the form of the induced effect, takes over from the individual nonplanar resolutions of individual orientation disparities.

Global vertical disparity, together with its associated orientation disparities, arises ecologically from any close planar surface that is not equidistant from the two eyes. This is the case when the surface is in an eccentric position relative to the median plane. Realizing this, Ogle $(1938,1939)$ sought an explanation of the induced effect in the stereoscopic geometry that applies under conditions of asymmetric convergence. However, as will be made clear in what follows, the geometry applies whether or not the eccentric surface is converged upon.

\section{The Induced Effect and Stereoscopic Geometry}

Eccentrically positioned surfaces-that is, surfaces laterally placed relative to the median plane-normally would have both vertical and horizontal disparity introduced by the difference in the distances of the surfaces 
from the two eyes. This horizontal disparity adds to or subtracts from the horizontal disparity created by a slant of the surface relative to the frontal plane. This means that an appropriate slant response to horizontal disparity requires scaling to remove the component that results from the different distances of the surface from the two eyes (eccentricity of the surface). Vertical disparity provides a good index of surface eccentricity and is therefore a candidate for elicitation of the appropriate scaling of the depth response to horizontal disparity. Inappropriate elicitation of such scaling by the optical introduction of vertical disparity in noneccentric surfaces can account for the induced effect. The various theories based on this idea are reviewed by Gillam and Lawergren (1983). Mathematically explicit versions were independently developed by Longuet-Higgins and Mayhew (1982) and Gillam and Lawergren (1983). A feature of both of these theories was to show that multiple vertical disparities are capable of mathematically specifying the absolute distance of a surface as well as its eccentricity. Since the depth responses to horizontal disparities must be scaled for distance as well as eccentricity, these theories give vertical disparity a central role in the achievement of stereoscopic depth constancy at close distances.

The present study was an attempt to examine the empirical evidence for such a role through investigation of the classical induced effect. This effect occurs when vertical magnification is imposed on one eye's view of a frontal plane surface that is centered on the median plane and thus has no overall horizontal disparity. Optical vertical magnification of one image of such a surface exactly mimics the changes in vertical disparities that would be created by shifting the surface to a more eccentric position in the visual field but maintaining the same distance; that is, it alters the intercept but not the slope of the vertical disparity function (Gillam \& Lawergren, 1983). Thus there is a good geometric basis for supposing that vertical magnification of one eye's view would have the same

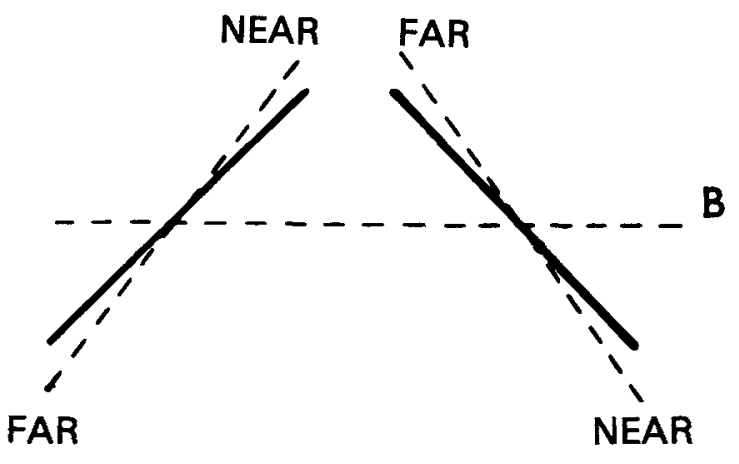

Figure 1. The left (unbroken line) and right (broken line) images of two planar oblique lines magnified vertically in the right eye. The apparent depths predicted on the basis of local horizontal disparities or local orientation disparities are shown. (From "The Induced Effect, Vertical Disparity, and Stereoscopic Theory" by B. Gillam and B. Lawergren, 1983, Perception \& Psychophysics, 34, p. 123. Copyright 1983 by the Psychonomic Society.)



Figure 2. An illustration of the fact that in eccentric positions in the visual field, equal horizontal angles are subtended at the two eyes by a slanted plane ( $\left(s^{\prime}\right)$, whereas in a central position, the same horizontal angles are subtended by a frontal plane (ff').

effect on the scaling of horizontal disparity as would placing the surface eccentrically. The critical point in accounting for the induced effect is that for a surface that is viewed symmetrically by the two eyes, zero horizontal disparity is consistent with a frontal plane position, whereas for a surface that is eccentric to have zero horizontal disparity, it must be a slanted surface (see Figure 2). Figure 3 shows the relationship between vertical disparity, eccentricity, and slant. The circles represent loci of constant vertical disparity. Each radial line represents an angular eccentricity relative to the median plane. The label on each of these lines (twice the angular eccentricity value) gives the surface slant consistent with zero horizontal disparity at that eccentricity; this represents the predicted induced effect at that eccentricity. To derive the eccentricity from vertical disparity, information about distance along the median plane is also required. For close distances, this is specified by the gradient, or the rate of change of vertical disparity across a surface (Gillam \& Lawergren, 1983).

\section{The Role of Conflict in the Induced Effect}

One factor that must be taken into account in any consideration of the induced effect is information conflict (Gillam \& Lawergren, 1983). It is known that the stereoscopic slant response to a given horizontal disparity is influenced by oculomotor information about both distance and eccentricity in the visual field (Ebenholtz \& Paap, 1973; Wallach, Gillam, \& Cardillo, 1979). This suggests that the necessary scaling of horizontal disparity in normal viewing is at least in part achieved by monitoring oculomotor responses. Under normal conditions, distance and eccentricity signaled by visual infor- 


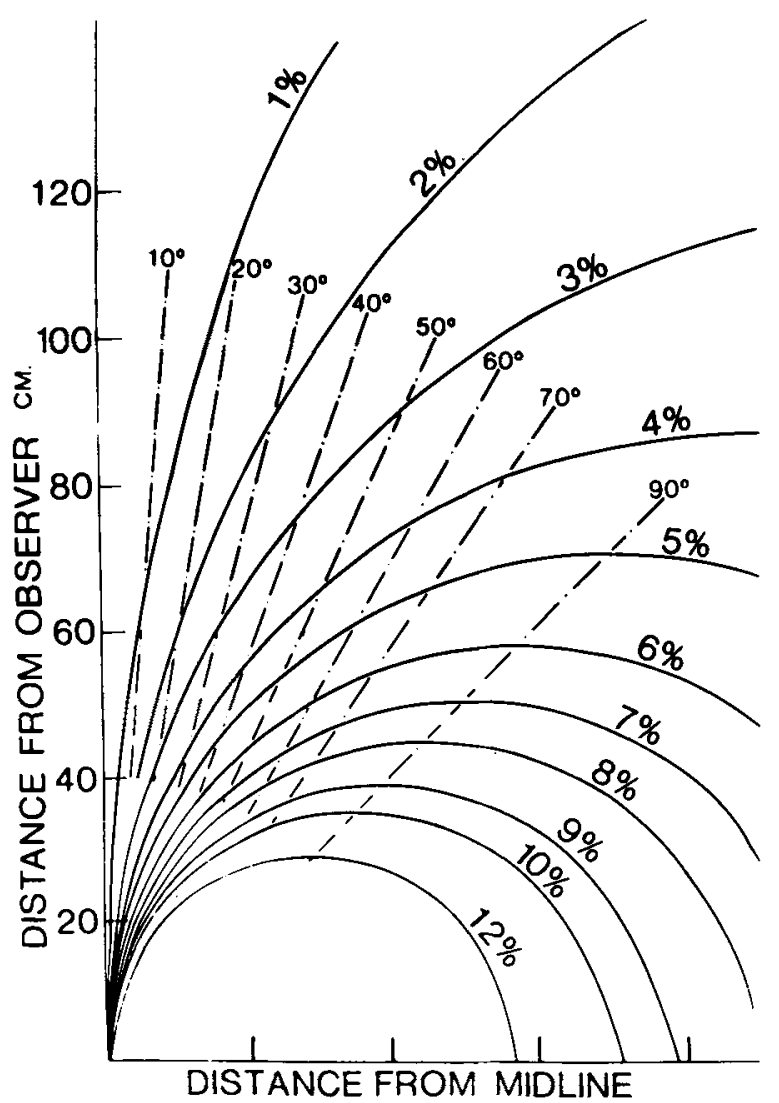

Figure 3. Scale diagram showing the loci (circles) that would result in various percentage magnifications of the right-eye image relative to the left (from $1 \%$ to $12 \%$ ). The radiating lines represent eccentricities. The label on each line represents the slant that would give rise to zero horizontal disparity at the eccentricity value of that line. It is twice the angle of eccentricity (see Gillam \& Lawergren, 1983). The $x$-axis represents distances along the frontal plane, and the $y$ axis represents distances along the median plane, with a point midway between the eyes as origin. Both axes have the same scale. (From "The Induced Effect, Vertical Disparity, and Stereoscopic Theory" by B. Gillam and B. Lawergren, 1983, Perception \& Psychophysics, 34, p. 126. Copyright 1983 by the Psychonomic Society.)

mation (vertical disparity) complement the oculomotor information. In the case of optically induced vertical disparity, however, there is conflict instead, since visual information signals eccentricity in the visual field, whereas oculomotor information places the surface centrally. The strong ocular discomfort associated with the induced effect suggests that conflict between the visual and the oculomotor systems is indeed present. Conflict within the visual system does not cause discomfort. For example, no discomfort is associated with viewing through a pseudoscope.

\section{Predicted Magnitude of the Induced Effect}

Even if it is accepted that the induced effect results from a rescaling of the slant response to horizontal disparity, triggered by the vertical disparity present, there is some question about what magnitude of slant would be predicted. Theories differ, and the reasons are worth going into because they reflect a difference in the fundamen- tal assumptions about just what slant is given visually under normal circumstances. Ogle (1950), Longuet-Higgins and Mayhew (1982), Frisby (1984), and Koenderink and van Doorn (1976) assume that stereoscopic information gives slant (relative to the normal) to the direction of gaze. The slant of the normal relative to the frontal plane of the head is presumably known by the visual system for each direction of gaze (indeed, it is the same as the angle of gaze; Gillam \& Lawergren, 1983). This slant must be added to the stereoscopically given slant to achieve geographical slant. If this were not done, the slant of a surface would change each time the eyes moved. Gillam and Lawergren (1983), on the other hand, assume that stereoscopic information is used to derive slant relative to the frontal plane of the head, so that stereoscopic slant equals geographical slant for a given head position regardless of direction of gaze. The normal does not have to be known. Their theory has the advantage that it does not distinguish between the case in which the eyes are actually converged asymmetrically and the case in which the eyes are looking straight ahead and the slanted surface is in an eccentric position. Both horizontal and vertical disparities are regarded as being present in the optic arrays of the two eyes regardless of direction of gaze.

The predicted induced effect (i.e., the slant required to produce a zero horizontal disparity at the eccentricity and distance indicated by vertical disparity) will be equivalent to the geometric effect if slant is assumed to be given stereoscopically relative to the normal, and twice the geometric effect if it is given relative to the frontal plane (Gillam \& Lawergren, 1983).

\section{The Asymptote of the Induced Effect and a Postulated Relationship to Observation Distance}

The induced effect was measured as described in a series of papers by Ogle $(1938,1939)$. He found that the geometric and induced effect were of the same magnitude but opposite in sign up to a magnification value of around $6 \%$, at which point the induced effect reached an asymptote and the geometric effect continued to increase up to approximately $12 \%$ magnifiction. The lowmagnification asymptote for the induced effect has always been difficult to explain. However, reference to Figure 3 shows that at $40 \mathrm{~cm}$, which is the distance Ogle used, vertical disparities beyond about $6 \%$ do not occur. It appears possible, therefore, that the asymptote might represent a failure to respond to ecologically impossible stimulus conditions. If so, the asymptote will move to lower magnification values as observation distance increases. At $100 \mathrm{~cm}$, for example, vertical disparities beyond $2 \%$ do not occur (see Figure 3). The only evidence from Ogle's work concerning this point is a plot of the data from one subject at two distances, 20 and $70 \mathrm{~cm}$ (Ogle, 1939). At $20 \mathrm{~cm}$ the maximum induced effect was at $10 \%$ and $12 \%$ for right- and left-eye magnifications, respectively, whereas for the $75-\mathrm{cm}$ distance it was at $8 \%$ and $6 \%$. These results were obtained using a nulling technique.

In the following experiments, we measured the induced effect and the geometric effect as functions of both mag- 
nification and observation distance. These measurements served in particular to test the hypothesis that the asymptote varies with observation distance (as predicted by the limits of Figure 3 ) and in general to investigate the scaling of the magnitude of the induced effect with distance. This has not been systematically investigated before.

\section{Nulling as a Technique}

Ogle's $(1938,1939)$ data were obtained by having subjects move the viewed surface so that it appeared to be in a frontal plane. The slant induced by vertical magnification of one eye's view was nulled out. We considered it important to use a matching technique instead, for the following reasons: Nulling does not measure a perceptual effect, but rather the conditions necessary for its elimination. Obviously, this will have some theoretical significance of its own, but it will not be the same as a measure of the perceptual effect itself. Insofar as nulling measures of the induced effect show that a horizontal disparity of the same magnitude as the vertical disparity, but with the opposite sign, are required to eliminate the induced effect, we may conclude that the induced effect occurs whenever an imbalance between these two disparities occurs. We may conclude nothing, however, about the magnitude of the (slant) response to that imbalance, nor may we conclude that the induced effect and the geometric effect are necessarily of the same magnitude in their perceptual effects because they require equal amounts of horizontal disparity to null them.

\section{EXPERIMENTS 1 AND 2}

\section{Method}

Subjects. In Experiment 1 there were 2 subjects, B.G. (the senior author) and D.C. (who was naive with respect to the induced effect). (The data for the subjects were obtained at different times, and only three lens magnifications and three distances were used for D.C., compared with four for B.G.) D.C. also served in Experiment 2 as did a new naive subject, N.C. All subjects were tested and found to be emmetropic at the time of testing.

Apparatus. The stimulus pattern was constructed to resemble Ogle's $(1938,1939)$ as far as possible and consisted of a sheet of plate glass $2 \mathrm{~mm}$ thick on which dots of $1 / 3 \mathrm{~cm}$ in diameter were painted at a pseudorandom density of 64 per square $5 \mathrm{~cm}$. (Ogle, 1938, 1939, did not specify the dot density he used.) Following Ogle, half the dots (selected at random) were painted on one side of the glass plate and half on the other. The dots were seen against an evenly illuminated light box placed behind the glass on which they were painted. The luminance of this background was $1,700 \mathrm{~cd} / \mathrm{m}^{2}$. An aperture at the subject's eyes held the appropriate lens and restricted the subject's field of view. The horizontal visual angle subtended by the pattern was $42^{\circ}$, and the vertical visual angle was $26^{\circ}$. These values were kept constant across changes in viewing distance by adjustments of binocular screens and septum. The frame holding the glass sheets was not visible.

The method of measurement was the one described by Gillam (1968): a comparison stimulus is presented in a monocular part of the visual field, and the subject must adjust this stimulus to appear parallel to the binocularly viewed dotted surface. In the present case, the lower part of the field was monocularly viewed and contained the comparison stimulus, which was a circular Meccano pulley wheel with oblique holes. This had been found in previous work to be an effective monocular comparison stimulus. The wheel had a diameter of $7.5 \mathrm{~cm}$ and was placed $8 \mathrm{~cm}$ in front of the glass below the dots, but seen against the same white background. Its height could be adjusted so that its top was always just below the bottom level of the dots, and it could be occluded for one eye without occluding any dots. At the closest viewing distance $(30 \mathrm{~cm})$, a smaller (5-cm-diameter) Meccano wheel was substituted. The comparison would have appeared as a silhouette against the light box except that it was also illuminated from in front to allow its details to be used for monocular slant information. (See Figure 4 for the subject's view of the apparatus.)

Afocal magnifiers, as described by Ogle (1950), were used for magnification.

Procedure-Experiment 1. In Experiment 1 the lens was always placed over the subject's right eye, and the comparison stimulus was, because of the arrangement of screens, visible only through the left eye. There were two sessions at each of four distances $(100$, 70,45 , and $30 \mathrm{~cm}$ ) presented in random order. During each session (at one distance), the subject was presented twice with each of eight conditions: the two lens axes (horizontal and vertical, for assessment of the geometric and induced effects, respectively) were combined factorially with the four lens magnifications. The 16 measurements were independently randomized for each session. After eight sessions (two at each of four distances) were completed, the entire series was repeated with the distances in the opposite order. This meant that in all there were 8 measurements of each distance $x$ lens axis $\times$ lens magnification condition.

Before each set of 16 measurements in a particular session, the subject was required to adjust the comparison stimulus to a series of six real slants seen binocularly so that we could check on the validity of the comparison stimulus as a slant measure. It was found that the subject was accurate to within about $3^{\circ}$ at all distances.

When instructed by the experimenter, the subject opened his/her eyes and viewed the dots for $30 \mathrm{sec}$ before beginning his/her adjustment of the comparison stimulus when signaled by the experimenter. The 30-sec viewing period allowed time for development of the effects of the lens. It is known that the latency can be considerable (Gillam, Flagg, \& Finlay, 1984; Seagrim, 1967). No time limit was set for making the adjustment, and bracketing was allowed. At the end of each trial, the comparison stimulus was set back to zero by the experimenter. The results of Experiments 1 and 2, since they are similar, are given together.

Procedure-Experiment 2. In Experiment 1, the geometric effect and the induced effect were both measured with magnification



Figure 4. The subject's view of the binocular surface and the monocular comparison stimulus used in the experiments reported. 
of the subject's right eye. This meant that the two effects were always in the opposite direction, and differences between the effects were confounded with any left-right asymmetries in the settings of the comparison device (although we knew from the practice settings that these were not large). In Experiment 2, both eyes were exposed to both horizontal and vertical magnifications. As far as possible, we imposed equivalent magnitudes of vertical magnification on one eye and horizontal on the other. Lenses were mounted around the circumference of two wheels, one for each eye. The appropriate lens was rotated into position before either eye by computer-controlled stepping motors that drove the wheels. Otherwise, the method and procedure were the same as in Experiment 1 with eight measures per point. In this experiment, the geometric effect and induced effect slants were in the same direction, and differences between them were confounded with differences between eyes. A similar result in both experiments would rule out as the critical factor either a bias due to slant direction or a bias due to eye.

\section{Results}

The results of Experiment 1 are given in Figure 5, which shows geometric effect (A) and induced effect (B) functions, with distance as the parameter, for B.G. (top) and D.C. (bottom). Figure 6 shows the two effects for N.C. in Experiment 2. It is clear that the geometric effect shows the predicted scaling with distance, whereas the induced effect does not. The results did not vary depending on the method used. This fact allows us to conclude that the difference between the effects is not owing either to the eye receiving magnification or to the direction of slant.

The data for all subjects show that at all distances the induced effect fell below the function predicted by Gillam and Lawergren (1983) at magnifications of $2 \%$ and

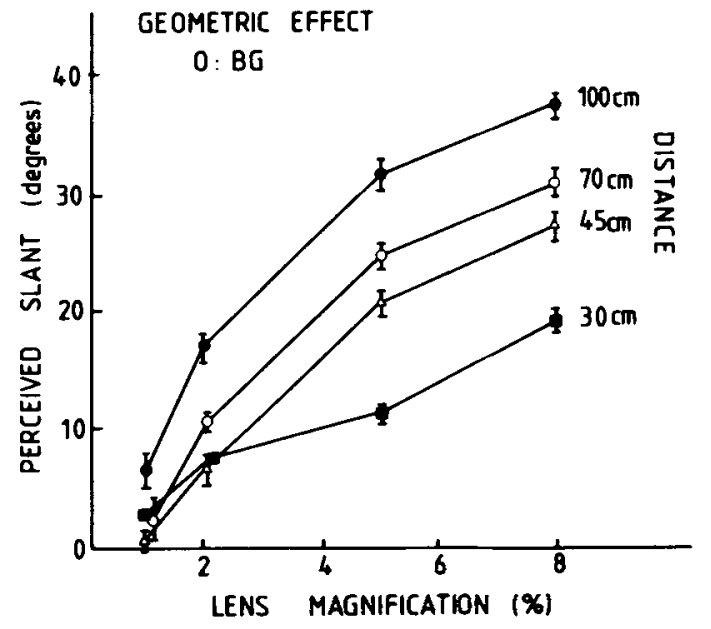

A

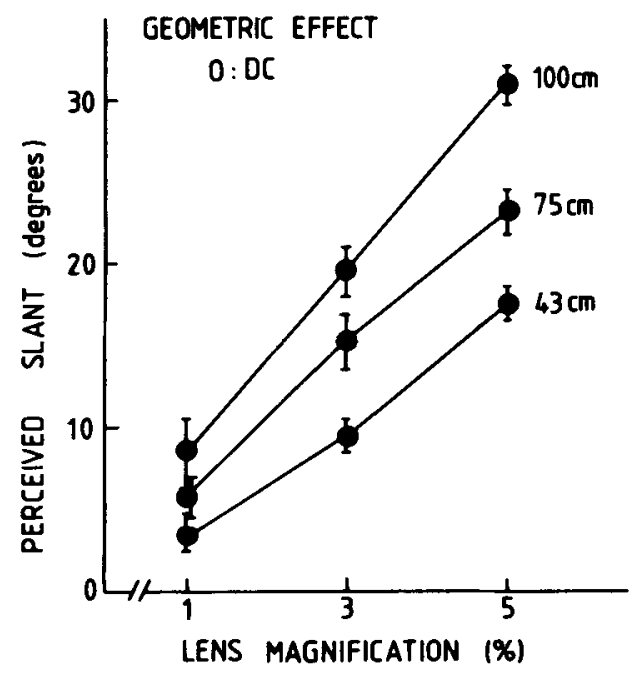

A

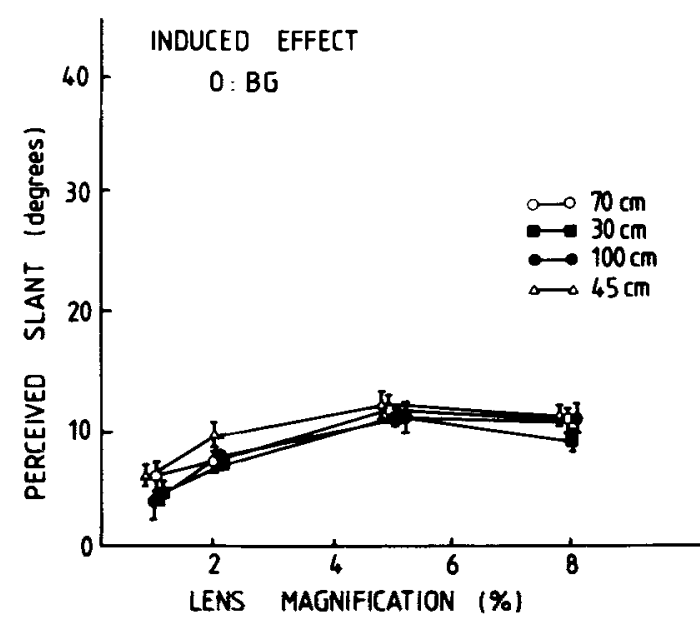

B

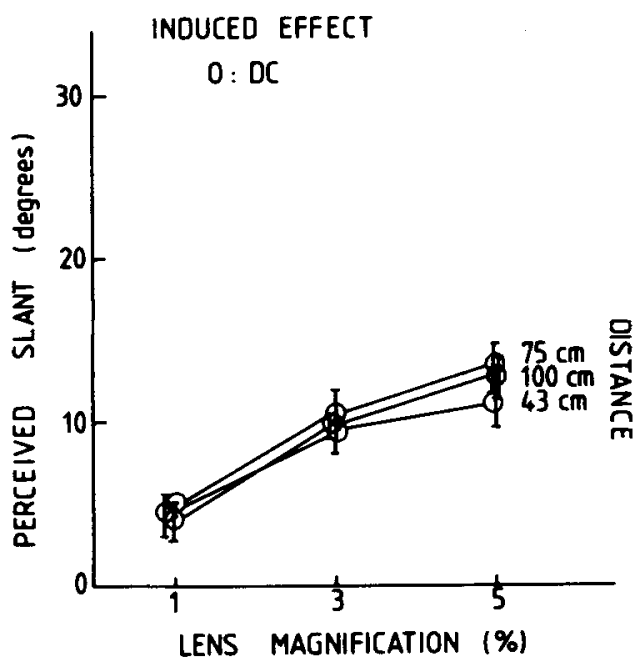

B

Figure 5. Functions for the geometric effect and the induced effect obtained in Experiment 1, plotted separately for Subjects B.G. (top) and D.C. (bottom). Distance is the parameter in each case. Standard errors are shown. These results were obtained under conditions in which the geometric and the induced effects produced slants in the opposite direction. 



Figure 6. Geometric and induced effect functions for Subject N.C. in Experiment 2, with distance as the parameter in each case. Standard errors are shown. These results were obtained under conditions in which the two effects produced slants in the same direction.

above. This represents a great attenuation of slant from the predicted value. To make this effect clear, the induced effect and the geometric effect are shown in Figure 7, plotted together for 1 subject. The induced effect also falls below the geometric effect at $8 \%$ for the $30-\mathrm{cm}$ distance, at $5 \%$ for the $45-\mathrm{cm}$ distance, at $3 \%-4 \%$ for the $70-\mathrm{cm}$ distance, and at $2 \%$ for the $100-\mathrm{cm}$ distance. These deviation values were the same across subjects and experiments. It should be noted that the divergence of the two effects occurs at a lower magnification than the maximum vertical magnification that would occur ecologically at each distance (see Figure 3).

The standard errors for the induced effect are no greater than for the geometric effect, and the results therefore presumably reflect a reliable perception of slant.

Although the magnitude of the induced effect is but a pale reflection of what would be expected were visual information alone sufficient to specify eccentricity, it should be borne in mind that the latter is also influenced strongly by oculomotor indicators.

Support for Gillam and Lawergren's (1983) theory, which predicts an induced effect greater than the geometric effect, comes from the strong suggestion in our data that the induced effect is greater than the geometric effect at very small magnifications, at which conflict with oculomotor indicators of eccentricity is minimal. Ogle (1938) stated that under certain conditions the induced effect can be more sensitive than the geometric effect, although this is not apparent from his data. For B.G. in Experiment 1 , the induced effect was significantly greater than the geometric effect with the $1 \%$ lens at three of the four distances. For D.C. it was only greater at the $30-\mathrm{cm}$ distance, and this was not significant. However, the induced effect was never significantly less than the geometric effect for either subject at $1 \%$. In Experiment 2, in which the two effects were perhaps more directly comparable in that they were represented by slants in the same direction (with lenses on different eyes), the greater magnitude of the induced effect than of the geometric effect at small magnifications was even more evident. The induced effect was greater than the geometric effect at three out of four distances for D.C., and at all distances for the $1 \%$ condition for N.C. In Experiment 2, we also measured the induced effect with a $0.5 \%$ lens. Because we only had one of these lenses, the geometric effect could not be measured at the same magnification; however, the induced effect for this low-magnification lens was significantly greater than the predicted geometric effect for two distances for D.C. and for three distances for N.C. In the other cases, the effect was not significantly different from the predicted geometric effect. It was never significantly lower. For the $0.5 \%$ magnification, the induced effect was as great in magnitude as predicted by Gillam and Lawergren.

The falloff in magnitude of the induced effect relative to prediction at greater magnifications may be attributed to conflict between eccentricity as specified by vertical disparity and as specified by convergence. To test this hypothesis, we plotted for subject B.G. the ratio of obtained to predicted induced effect as a function of the angle of asymmetric convergence specified by the vertical disparities present (equivalent convergence angle). Since the actual asymmetric convergence angle is zero, we assumed that the greater the equivalent convergence angle, the greater the conflict. All data are shown on a single graph (Figure 8). The ratio of obtained to predicted induced effect declines exponentially with the increasing equivalent convergence angle as given by vertical disparity, at least for the $30-\mathrm{cm}$ and $45-\mathrm{cm}$ points. This is assumed to be the result of increasingly strong counterindications to the vertical disparity eccentricity factor from extraretinal convergence signals. At the greater distances $(70$ and $100 \mathrm{~cm}$ ), an additional factor seems to be acting to attenuate the induced effect. This factor could be the relative insensitivity of vertical disparity as an index of eccentricity at greater distances.

\section{EXPERIMENT 3-NULLING METHOD}

Our results markedly disagreed with Ogle's (1938, 1939) concerning the magnitude of the induced effect. To 

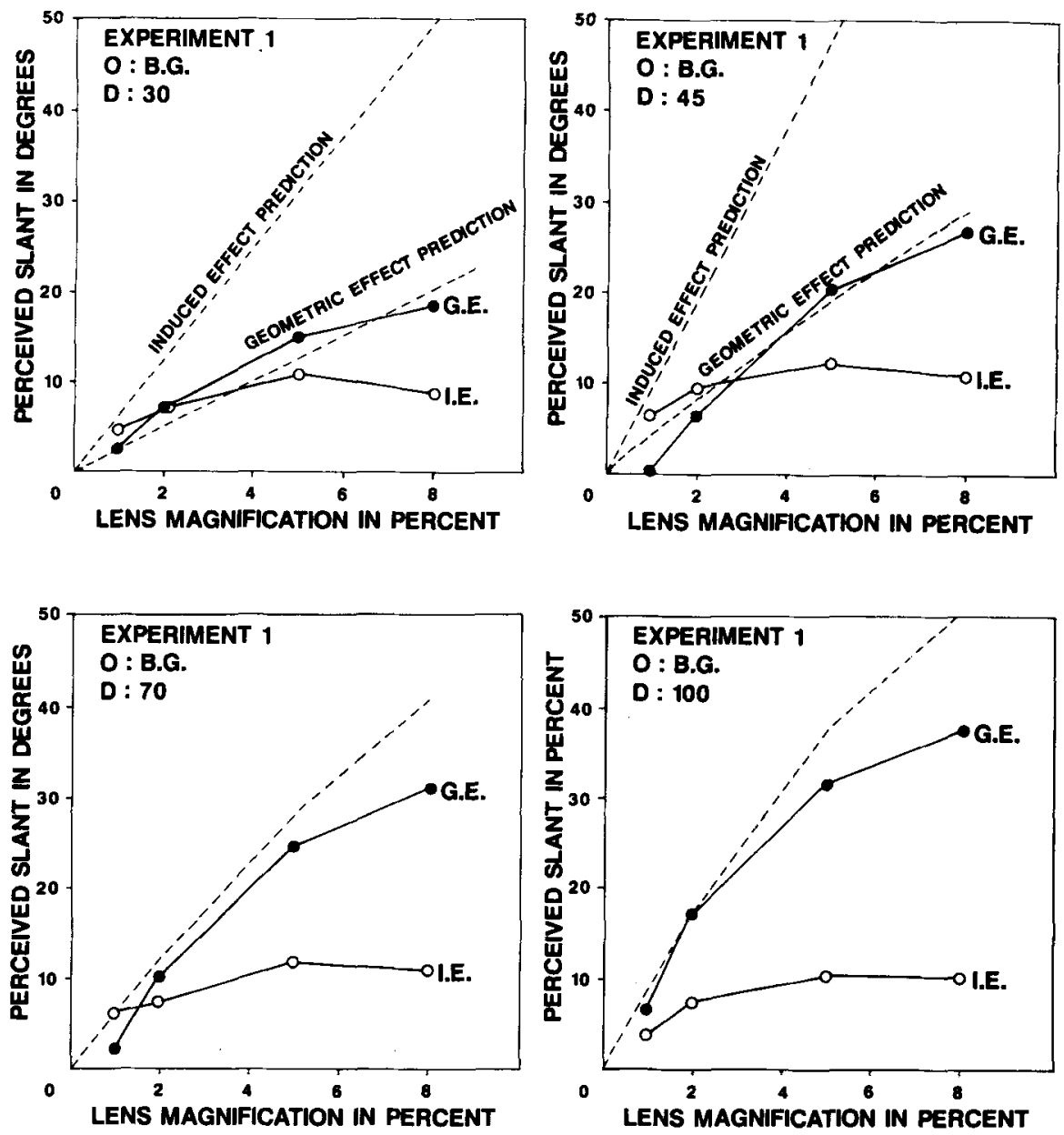

Figure 7. Induced effect and geometric effect data plotted together on the same graph as a function of magnification for each of four distances for Subject B.G. (Experiment 1). The dotted lines on the lower two graphs indicate geometric effect prediction.

see if we could obtain Ogle's results with our apparatus using his nulling technique, we carried out a subsidiary experiment on 2 naive subjects and the senior author, using a distance of $40 \mathrm{~cm}$ and magnifications ranging from $2 \%$ to $8 \%$ in the right eye. Both geometric and induced effects were measured for the same stimulus viewed under the same conditions as in our previous experiments, with eight measurements per data point. The subjects were required to move the frame holding the glass by pulling two strings attached to its sides until it appeared to be in the frontal plane. The starting position of the glass was always the physical frontal plane; no bracketing was allowed. Previous attempts in our laboratory to measure the aniseikonic geometric effect using nulling showed that it was very difficult for naive subjects, who tended to be distracted by the apparent expansion motions of the dots on the surface. These are not the motions that would normally accompany slant changes. The same difficulties occurred in making the present measurements. We found that 2 of the 3 subjects gave shallow, nonmonotonic functions for the geometric effect and, although they interestingly produced greater slants for the induced effect, they stated that they were not satisfied that the surface ever appeared to be in the frontal plane. One naive subject, however, did give a geometric effect function close to prediction, and his data are given for both effects in Figure 9. They are remarkably similar to the data we obtained using the matching technique, even showing the significantly higher induced effect at $1 \%$ magnification and the divergence of the two effects at about $3 \%$ for the $45-\mathrm{cm}$ distance. We cannot therefore entirely attribute the differences between our data and Ogle's data to the method of measurement used.

\section{GENERAL DISCUSSION}

\section{Ecological Considerations}

The results of the three experiments reported here failed to confirm Ogle's $(1938,1939)$ finding that the induced effect is as great as the geometric effect up to approximately $6 \%$ magnification. We found the induced effect to be much smaller, except at magnifications below $2 \%$, where the induced effect was sometimes greater than the geometric effect. Furthermore, we did not confirm the 


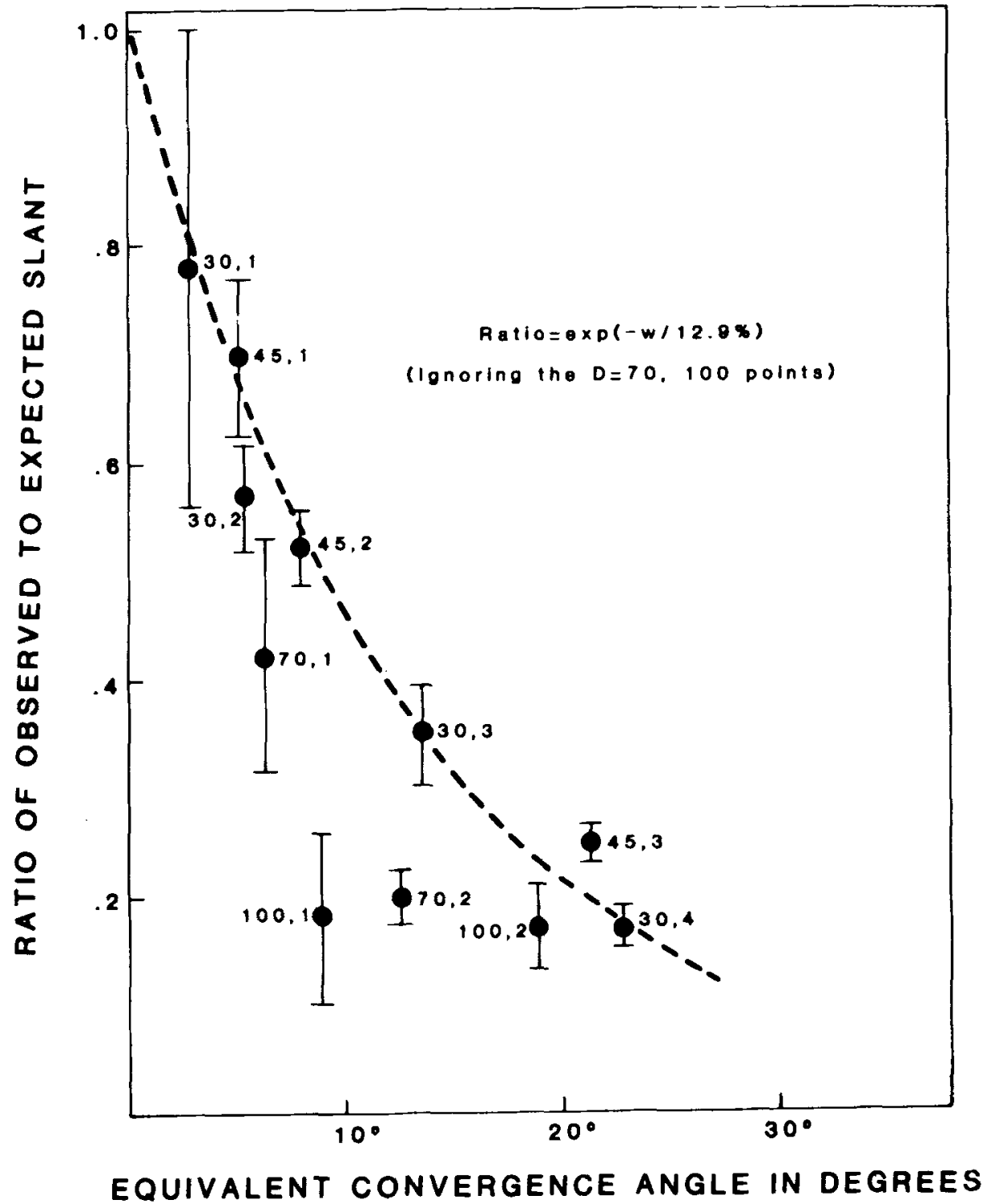

Figure 8. The ratio of obtained to predicted induced effect as a function of the asymmetric convergence specified by the vertical disparities present (equivalent convergence angle).

hypothesis we had formulated to account for Ogle's reported asymptote, namely that the effects of vertical magnification are limited to close distances where vertical disparity would occur naturally. On this basis, asymptotes at higher magnification values would be expected for closer observation distances, a prediction our data did not support. Further evidence against the idea that information provided by vertical disparity is discarded under observation conditions when it would not occur in real life is provided by the data obtained with $8 \%$ magnification. Significant magnitudes of induced effect at this magnification were found even at viewing distances of 75 and $100 \mathrm{~cm}$, well beyond the maximum distance at which such a magnification would occur in real life.

As mentioned earlier, Longuet-Higgins and Mayhew (1982) attributed the induced effect to an inappropriate registration of gaze angle rather than to registered eccentricity in the visual field. Assuming that eccentric gaze angles of more than $15^{\circ}$ would be unlikely to occur without a head movement, one might propose a further ecological limitation to the induced effect, in that magnification-distance combinations that correspond to gaze angles larger than approximately $15^{\circ}$ do not produce the effect. It can be seen from Figure 3 that on this basis the induced effect should not occur beyond $1 \%$ at $100 \mathrm{~cm}$, $2 \%$ at $70 \mathrm{~cm}, 3 \%$ at $45 \mathrm{~cm}$, and $6 \%$ at $30 \mathrm{~cm}$ (approximately). It can be seen from page 479 that these values do correspond quite well to the magnifications at which the induced effect deviates from the geometric effect (see Frisby, 1984). However, this approach does not explain the existence of an induced effect of significant magnitude for an $8 \%$ magnification at $100 \mathrm{~cm}$. Also, it should 


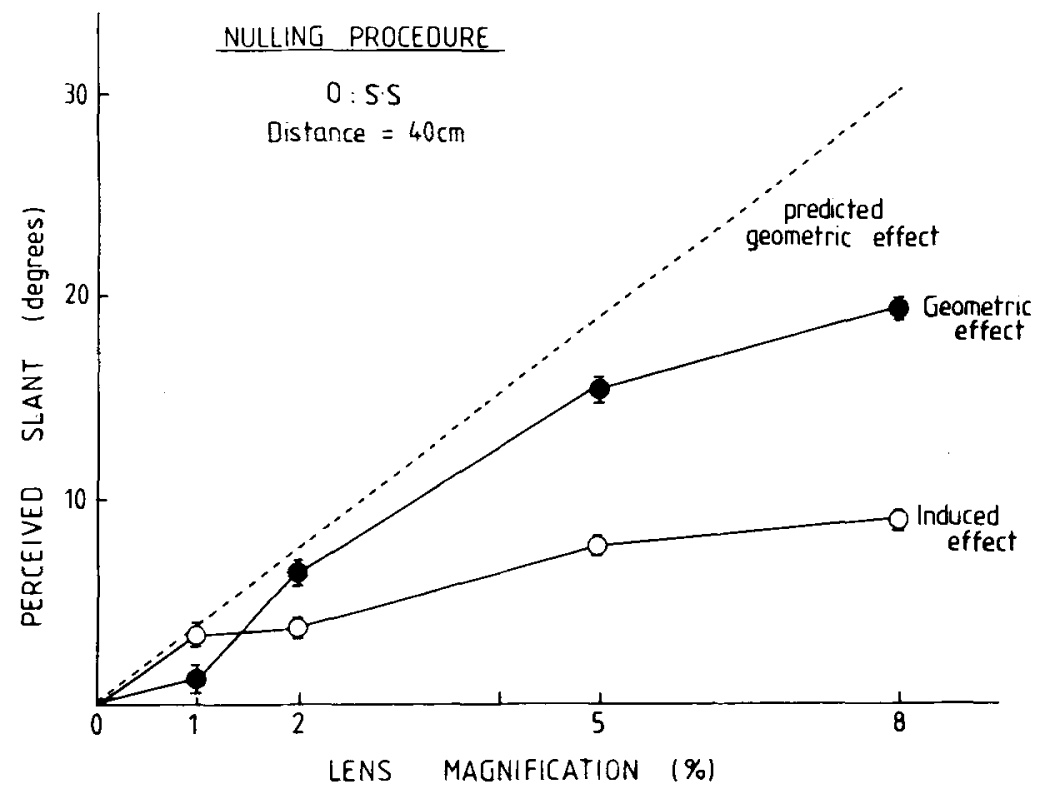

Figure 9. Induced effect and geometric effect as a function of magnitude obtained for 1 subject using Ogle's (1939) nulling method. Distance equals $40 \mathrm{~cm}$. Standard errors are shown.

be borne in mind that adequate stereoscopic scaling must be based on an index of eccentricity in the visual field rather than on gaze angle, since an eccentric surface will require appropriate scaling of horizontal disparity whether or not it is gazed at. Ideally, surfaces at different eccentricities should be processable at the same time, each according to the vertical disparity/horizontal disparity relationship present at that location. It could be that at eccentricities of $15^{\circ}$ or more a head movement rather than an eye movement results.

The presence of some degree of induced effect, even at those vertical magnifications and gradients that are too extreme to ever occur in real space, could mean that the mechanism for responding to vertical disparity operates without regard for the geometry of space perception. Alternatively, it could mean that qualitative aspects of the vertical disparity gradient, such as the presence of larger left (or right) eye images on both sides of the fixation point, bias the registration of eccentricity, even when the degree of disparity is too large for the viewing distance used.

\section{Conflict}

Vertical disparity anywhere may indicate eccentricity, but vertical disparity at the fixation point does indicate a sideward direction of gaze. In the case of the induced effect, this is in conflict with oculomotor indicators of where the eyes are pointing. This conflict may be responsible for the discomfort associated with the induced effect. As mentioned earlier, oculomotor information is also in general conflict with the eccentricity values specified by vertical disparities across the visual field. We have provided evidence (see Figure 8 ) that to some degree this

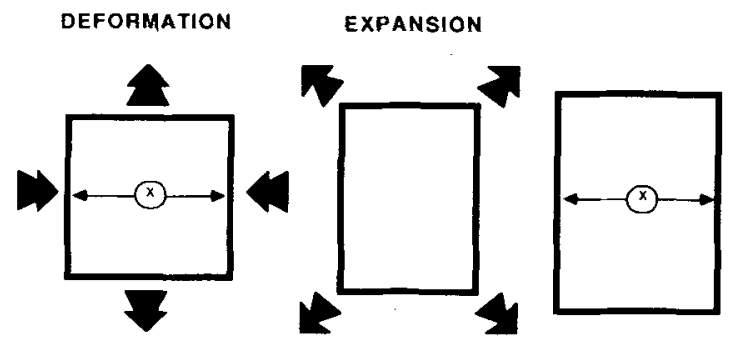

LEFT EYE VIEW

RIGHT EYE VIEW
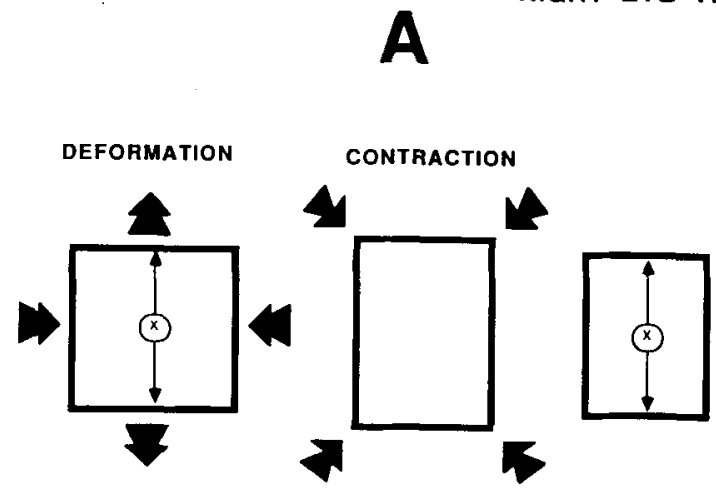

LEFT EYE VIEW

RIGHT EYE VIEW

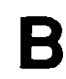

Figure 10. Showing that the same deformation (middle rectangles) will produce vertical disparity in favor of the right eye (A) or horizontal disparity in favor of the left eye (B). The difference between these two conditions is in an overall expansion in the vertical disparity case and an overall contraction in the horizontal disparity case. 


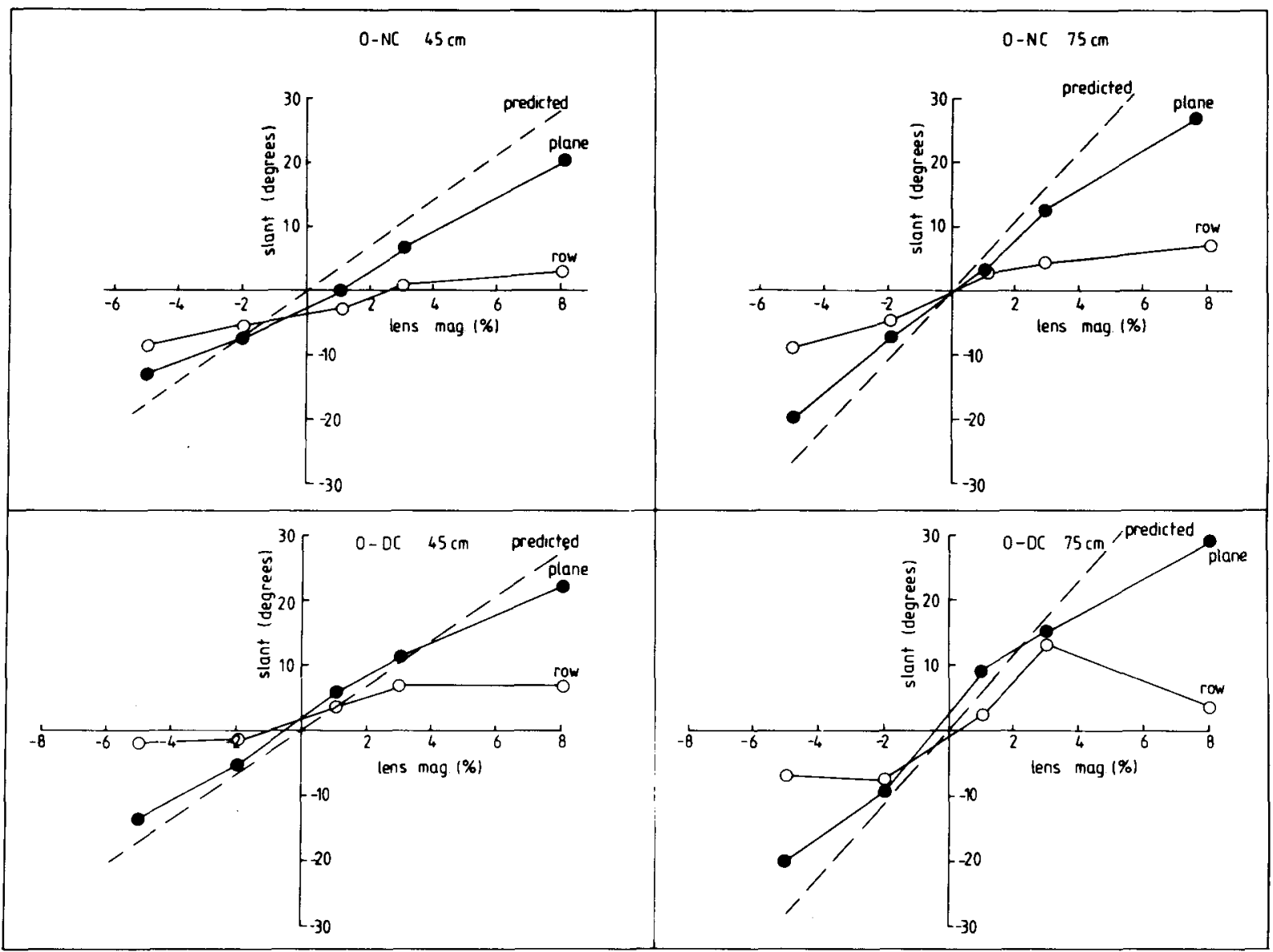

Figure 11. Geometric effect functions with vertical disparity present (plane) and without vertical disparity present (row).

conflict explains why the effect of vertical disparity is not as great as predicted by eccentricity theories of the effect.

Another possible factor needs to be considered for all distances. Since greater slants are predicted for greater eccentricities, it could be argued that it is the increasingly contradictory perspective slant information that causes the deviation of the induced effect from prediction at greater magnification. This is unlikely, however, since the geometric effect is also subject to this conflict and shows no effect of it except at $8 \%$ magnification. Random dots provide only very weak perspective information about slant.

\section{Direct Theories}

It would be very attractive to explain stereoscopic slant perception by invariants in the disparity field without necessarily referring specifically to horizontal and vertical disparities. This approach has been adopted by Koenderink and van Doorn (1976), who proposed that both the geometric and induced effects are responses to the deformation component of the transformation between left- and right-eye views. Deformation refers to an expansion of the image along one dimension accompanied by a proportional contraction along the orthogonal dimension. Nakayama (1985) listed deformation as the invariant for slant perception in the case of motion parallax. As can be seen from Figure 10, the deformation component is equivalent for horizontal minification of an image and vertical magnification of the same image. Since minification of the right image is formally equivalent to magnification of the left image, this theory would account for the qualitative similarity of the geometric and induced effects and must be regarded as an interesting and promising analysis of the problem.

However, such an approach is subject to the general problem of postulating direct computation of slant from entirely visual indices, such as horizontal and vertical disparities, without postulating an explicit representation of distance and eccentricity. The essential problem is how to give an account of the manner in which oculomotor information is incorporated into the system? If the deformation component directly gives slant, why the very large difference in magnitude of the induced and geometric effects? Also, why the well-documented discomfort of the induced effect but not of the geometric effect? In the case of vertical magnification, in addition to the deformation component, there is a dilation of the magnified image. These two together make up the vertical magnification. The dilation component could conceivably give rise to a 
registered eccentricity in conflict with oculomotor indications (Rogers \& Koenderink, 1986), thus accounting for the discomfort. However, a similar dilation component (actually a contraction) is required to complete the description of a horizontal disparity when the deformation component is extracted. Yet the geometric effect does not produce discomfort. A limitation of deformation is that it is not invariant with distance and would therefore require some other factor to scale the response to it. The great advantage of the Longuet-Higgins and Mayhew (1982) and of the Gilliam and Lawergren (1983) approaches is that they show that vertical disparity can completely specify the spatial parameters necessary to scale horizontal disparity, including distance (at least for distances up to around a meter). Given that the induced effect does not appear to alter with distance, this could be regarded as a moot point. However, the geometric effect, which is also a response to deformation according to Koenderink and van Doorn (1976), does scale with distance (at least for close distances). This cannot be explained by deformation alone, whereas it can be explained by disparity gradients.

\section{Conclusions}

In summary, our measurements of the induced effect do not give strong support to any current theory of the effect, although they are not inconsistent with eccentricity theories, given the assumption that conflict with oculomotor information will reduce the registered eccentricity from the value indicated by purely visual information. Support for the theory is much stronger if we add the assumption that vertical disparity is used only to scale horizontal disparity at small eccentricities and near distances. Independent support for the idea that vertical disparity is used to scale the slant response to horizontal disparity is given by a preliminary finding that the geometric effect shows poor scaling with both horizontal magnification and distance when the vertical dimension is eliminated. We found this by comparing the geometric effect for a horizontal row of dots and an entire field of dots subtending the same horizontal visual angle. The dot row was at the subject's eye level with a density of 10 points per inch. Geometric effect measurements on this stimulus were originally collected as pilot data in Experiment 2, immediately following the whole field measurements at
45 and $70 \mathrm{~cm}$. The single row results can therefore be compared with the whole field results obtained at the same distance. Even though there were only four measurements per point, the results were so clear-cut that they are given here in Figure 11. The single row measurements are relatively flat, whereas the whole field results follow prediction. Clearly, when vertical disparity is eliminated, the quantitative response to horizontal disparity is poor.

\section{REFERENCES}

Arditi, A., Kaufman, L., \& Movshon, J. A. A: (1981). A simple explanation of the induced size effect. Vision Research, 21, 755-764.

Ebenholtz, S. M., \& PAaP, K. N. (1973). The constancy of object orientation: Compensation for ocular rotation. Perception \& Psychophysics, 14, 458-470.

FrISBY, J. (1984). Vision: An old illusion and a new theory of stereoscopic depth perception. Nature, 307, 592-593.

GiLLAM, B. (1968). Perception when stereopsis and perspective conflict: Experiments with aniseikonic lenses. Journal of Experimental Psychology, 78, 299-305.

Gillam, B., FlagG, T., \& Finlay, D. (1984). Evidence for disparity change as the primary stimulus for stereopsis. Perception \& Psychophysics, 36, 559-564.

Gillam, B., \& Lawergren, B. (1983). The induced effect, vertical disparity, and stereoscopic theory. Perception \& Psychophysics, 34, 121-130.

Koenderink, J. J., \& van Doorn, A. (1976). Geometry of binocular vision and a model for stereopsis. Biological Cybernetics, 21, 29-35.

Longuet-Higgins, H. C., \& MaYheW, J. E. W. (1982). A computational model of binocular depth perception. Nature, 297, 376-378.

Nakayama, K. (1985). Biological image motion processing: A review. Vision Research, 25, 625-660.

OGLE, K. N. (1938). Induced size effect: I. A new phenomenon in binocular vision associated with the relative sizes of the images in the two eyes. Archives of Ophthalmology, 20, 604.

OGLE, K. N. (1939). Induced size effect: II. An experimental study of the phenomenon with restricted fusion contours. Archives of Ophthalmology, 21, 604.

OGLE, K. N. (1950). Researches in binocular vision. New York: Hafner. Rogers, B., \& KoEnderinK, J. J. (1986). Monocular aniseikonia: A motion parallax analogue of the disparity induced effect. Nature, 322, 62-63.

Seagrim, G. N. (1967). Stereoscopic vision and aniseikonic lenses: I and II. British Journal of Psychology, 58, 337-356.

Wallach, H., Gillam, B., a Cardillo, L. (1979). Some consequences of stereoscopic depth constancy. Perception \& Psychophysics, 25, 235-240.

(Manuscript received February 3, 1987; revision accepted for publication June 3,1988 .) 